\title{
Application of Atom Probe Tomography in Understanding Thin Film Chemical Ordering and Stress Behavior
}

\author{
G.B. Thompson*, B. Fu*, D. Means*, and B. Wang* \\ * The University of Alabama, Department of Metallurgical \& Materials Engineering \\ Tuscaloosa, Alabama 35487
}

Thin films exhibit large surface-to-volume ratios, allowing interfacial thermodynamics to play a significant role in the microstrutural evolution. Atom probe tomography's (APT) ability to detect atoms of equal sensitivity with near atomic spatial reconstruction resolution makes it an ideal technique to quantify the interfacial chemistry in these films. For example, in multi-element thin films, preferential partitioning or intermixing of species can regulate stresses or phases by changing the relative thermodynamic energies at the interfaces. In this study, three particular cases have been characterized using APT in order to evaluate the role of atomic-level chemical structure on either the stress or phase stability in thin films.

Arguably, most work on in situ thin film stress evolution has been for elemental materials. While these results have been paramount in our basic understandings of thin film nucleation and growth, multicomponent systems have the added complexity of interactions between different atom types, such as segregation or chemical reactions. These interactions can lead to stress responses that deviate from single elemental materials. A series of $40 \mathrm{~nm} \mathrm{Fe}_{\mathrm{x}} \mathrm{Pt}_{1-\mathrm{x}}(0 \leq \mathrm{x} \leq 1)$ thin films have been sputter-deposited. Elemental Fe films exhibited a tensile stress state, indicative of low growth mobility. In contrast, elemental Pt films exhibited compressive stress post-coalescence indicting higher intrinsic growth mobility. By alloying the elements together, the stress state was tuned to be either tensile or compressive. Surprisingly, for some overall Fe-rich films, these films retained a compressive stress state. APT revealed that the grain boundaries for these films were enriched in Pt, Figure 1. For the zero stress state, the film's grain boundaries were not enriched with either species. The change in film stress has been explained by the preferential segregation of either the tensile or compressive characteristic species into the grain boundaries [1].

The next example involves the A1 to $L 1_{0}$ chemical ordering reaction in $\mathrm{Fe}_{52} \mathrm{Pt}_{1-\mathrm{x}} \mathrm{Ta}_{\mathrm{x}}$ thin films, where $\mathrm{x}=1.4$ or 7.3 at.\% [2]. There have been conflicting reports in the literature if Ta additions either lower or raise the ordering temperature. Our results demonstrated that Ta suppresses order. The APT data revealed that ordering would not commence until the matrix was depleted in Ta by the formation of clusters, Figure 2. For clusters that formed on the grain boundaries, they provided a Zener-pinning based mechanism which reduced the grain growth associated with the increased annealing temperatures necessary to bring about the phase transformation.

Finally, when nanolaminates of elemental materials are deposited as a multilayer, one or the other species can change its crystal structure or phase if its layer thickness is significantly reduced. This phase transformations have been described as a competition between the volumetric energy increase balanced by the interfacial free energy reduction associated with the change in phase. These phase transformations can be represented on a bi-phase diagram which plots the inverse bilayer spacing, $\lambda$, verses the volume fraction, $f_{\mathrm{i}}$, in a unit bilayer of the multilayer. Note the volume fraction multiplied with $\lambda$ yields the thickness for that particular 
layer. A series of $\mathrm{Ti} / \mathrm{Nb}$ multilayers were sputter deposited and at a specific volume fraction and bilayer spacing, the hcp Ti became bcc Ti [3]. APT revealed significant inter-diffusion of $\mathrm{Nb}, \mathrm{a}$ bcc or $\beta$-stabilizer, in the bcc Ti layers. When the Ti adopted the hcp structure, negligible $\mathrm{Nb}$ inter-diffusion into the Ti layer was found. The incorporation of $\mathrm{Nb}$ lowered the volumetric energy barrier for the hcp to bcc Ti phase transformation.

\section{References:}

[1] B. Fu and G.B. Thompson JAP 108 (2010) 043506

[2] D. Means, B. Wang, and G.B. Thompson JAP 108 (2010) 104910

[3] G.B. Thompson, R. Banerjee, S.A. Dregia, and H.L. Fraser J. of Materials Research 19(3) (2004) 707.

[4] G.B. Thompson recognizes the support of the National Science Foundation, under award DMR0529369, for the FePt based research.

(a)

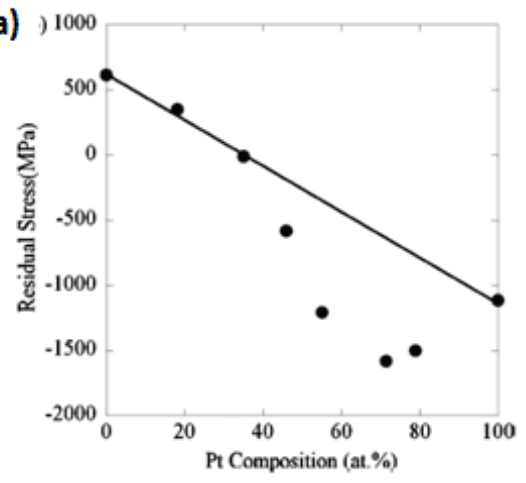

(b)

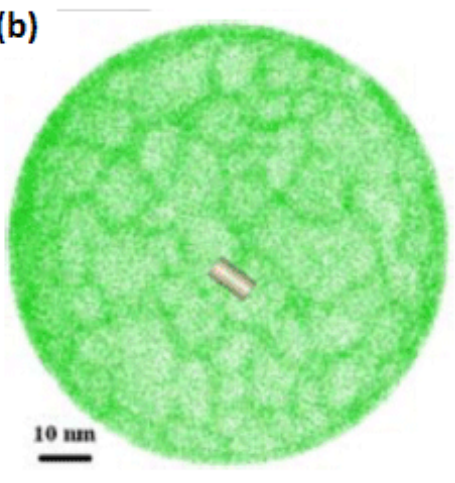

(c)

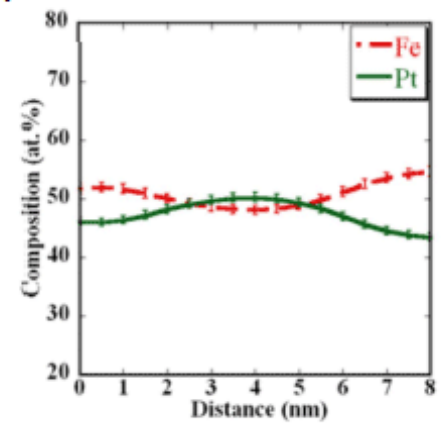

Figure 1: (a) Residual film stress at the end of $40 \mathrm{~nm}$ of growth for various compositions of $\mathrm{Fe}_{\mathrm{x}} \mathrm{Pt}_{1-\mathrm{x}}$ (b) $\mathrm{Pt}$ atom map of $\mathrm{Fe}_{55} \mathrm{Pt}_{45}$ which showed Pt-enrichment at the grain boundaries (c) 1D composition profile across a grain boundary. These images are taken from reference [1].

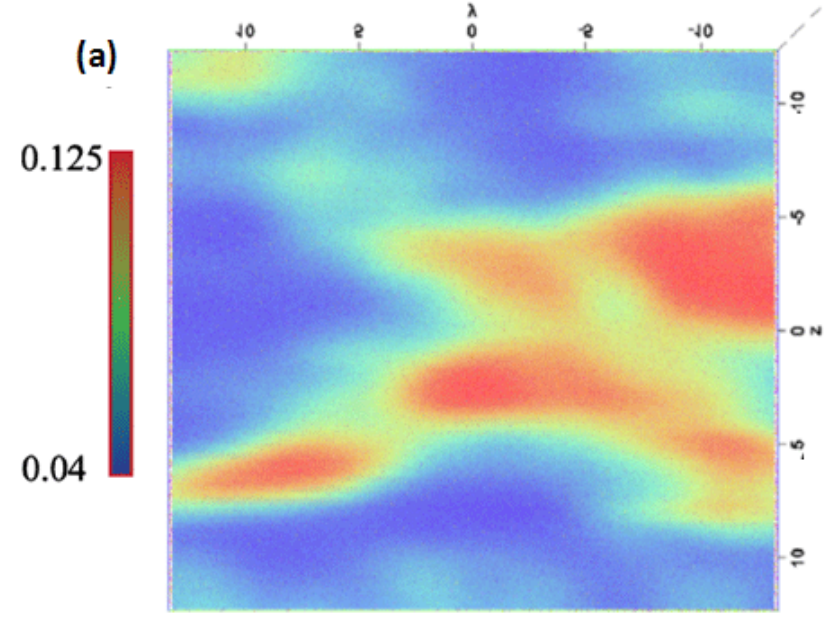

(b)

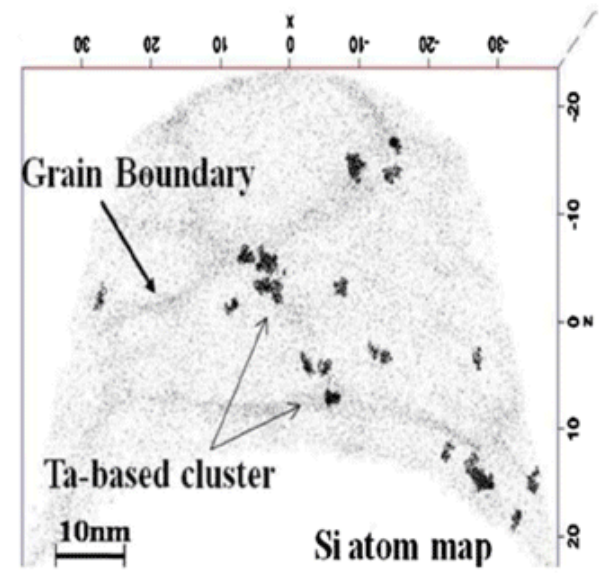

Figure 2: (a) 2D Ta concentration map for $\mathrm{Fe}_{52} \mathrm{Pt}_{40.7} \mathrm{Ta}_{7.3}$ annealed at $750^{\circ} \mathrm{C}$. Red regions represent higher Ta concentrations. (b) $\mathrm{Si}$ (impurity) atom map revealing the $\mathrm{Fe}_{52} \mathrm{Pt}_{40.7} \mathrm{Ta}_{7.3}$ thin film microstructure annealed at $750^{\circ} \mathrm{C}$. Note the presence of clusters near the grain boundaries which helped to reduce grain growth. These images were taken from reference [2]. 